\title{
Not enough traction: Barriers that aspiring researchers from low- and middle-income countries face in global health research
}

\author{
Constance S. Shumba ${ }^{1}$, Adelaide M Lusambili² (D) ${ }^{a}$ \\ 1 School of Nursing and Midwifery, Aga Khan University, ${ }^{2}$ Department of Population Health, Medical College, Aga Khan University \\ Keywords: language, gender, global health, Imics, disparities, researchers, barriers \\ https://doi.org/10.52872/001c.25802
}

Journal of Global Health Economics and Policy

Vol. 1, 2021

\begin{abstract}
There is a growing concern of low representation of researchers from low-middle-income countries (LMICs) in the publication of global health research in high-impact peer-reviewed journals. Nobody denies that researchers from the developing world generally face several obstacles to publishing their research. In this viewpoint, we share some of the barriers we have observed from our experience working in both academia and global health practice in low and middle-income countries such as limited opportunities for research funding, gender disparities, and language barriers. Beyond presenting the barriers, we also provide some pragmatic solutions to addressing these barriers through increased research financing, capacity building, gender equity and inclusion, and editorial support. Most importantly, we call for setting a new level of ambition in redressing the imbalances and actualizing the leadership and emergence of a veritable critical mass of LMICs researchers.
\end{abstract}

\section{INADEQUATE RESEARCH FUNDING AND CAPACITIES FOR LMICS INSTITUTIONS}

It is a known fact that a large amount of research published in global health originates from high-income countries (HICs), although the research projects often collect data from LMICs. ${ }^{1}$ Funding for this kind of research mainly originates from HICs, due to resource constraints and the lack of research prioritization in LMICs. Research financing in LMICs is markedly different from HICs, with several implications for generating high-quality evidence to inform policy and practice and the development of research expertise among LMICs researchers. The majority of LMICs' governments have historically failed to allocate meaningful resources for research and capacity-strengthening for academic and research institutions. Therefore, local universities that embrace a research culture need to collaborate with institutions from HICs to obtain funding. Unlike HICs, many LMICs students are only introduced to research towards the end of their first degrees when they have to conduct research projects before graduation. Due to the limited research career options, potential researchers may only implement research projects as a requirement for partial fulfillment of their degrees and not as a pathway to a defined career trajectory. Those who take research career paths are inadequately prepared and often require intensive mentoring by senior researchers. Only a few mentors at LMICs institutions are grounded in research meaning even where curricula include research methods there is a disso- nance with practice. Many of the LMICs' universities are not research-intensive and lack a critical mass of researchers and research mentors. Equally important, research internship opportunities for aspiring graduates are often limited. The few that exist are competitive, with many organizations opting to offer internship opportunities to students and graduates from HICs. This practice presents missed opportunities for capacity building of emerging LMICs researchers. In general, although the importance of research in guiding evidence-informed policies is recognized, there is often a disconnect between evidence generation and use. This disconnect results from poor investments in research, lack of timely evidence generation, and inability to translate the results into policy and practice.

The lack of funding support for research has meant that most local universities and non-governmental agencies have to seek funding from HICs. From our experience, researchers are hired for time-bound projects by partners in the global north, who leave after the end of the project with no time and money allocated for them to contribute to writing papers. Researchers working on specific projects have limited opportunities for continuity beyond the project lifecycles. Researchers from LMICs hired on these projects lack opportunities to contribute to the publications by principal investigators from HICs resulting in poor research career trajectories. Opportunities to be first or senior authors are often reserved for their HIC collaborators while they are mentioned in acknowledgments. ${ }^{1}$ Additionally, LMICs institutions often receive a minimal budget for implementing 
the research compared to their HICs partners due to salary disparities as researchers from HICs are generally highly paid. In addition to the lack of financial support from LMICs governments and academic institutions, the poor integration of research in the organizational cultures adds to the complexity. In the same vein, researchers from LMICs may not be interested in contributing their time to write papers for a career track that only offers temporary opportunities.

Our experience suggests some level of acceptance of the status quo among LMICs researchers. After all, our governments do not invest in research, ${ }^{2}$ and LMICs do not feel they have a voice, and 'He who pays the piper calls the tune'. Research funding institutions should develop a mechanism where partnerships must commit to equitably financing research in LMICs and building the capacity of LMICs researchers with equitable and extended support for publishing the results. Consequently, governments and research institutions from LMICs should also increase their investments in building the capacity of researchers and for research projects to respond to local research priorities. Organizations such as the Kenya Medical Research InstituteWellcome Trust are an exception and are worth emulating in research capacity strengthening. They provide internship opportunities to Kenyan graduates and have played a crucial role in developing some of the leading research scientists in the country. Further, more tools for reporting research should be used by journal editors to gauge whether the publication has included the key authors. For instance, in qualitative research reporting, we have the Consolidated criteria for reporting qualitative research (COREQ): a 32-item checklist for interviews and focus groups which should be embraced. ${ }^{3}$ More tools specific to different approaches should be developed by other disciplines to help journal editors provide guidance and evaluate submissions.

\section{GENDER DISPARITIES}

While there are gender disparities in global health research generally, the underrepresentation of women researchers from LMICs is even more pronounced. ${ }^{4,5}$ This gendered dimension is crucial as women researchers are not accorded similar opportunities as men and come from contexts with deep-seated patriarchal systems and gender inequalities. Women from LMICs still have caring responsibilities, and the playing field for entry and retention in research careers is not level and favors elitist men. In addressing these disparities, first, a greater focus on supporting women in research is required, with consistent access to training, mentoring, publication, and grant opportunities for women at all stages of their research careers. Targeted research leadership development programs can be run for women with publication and grant writing support and dedicated research funds for female researchers. Second, inclusiveness can incorporate ways to support women from the LMIC to make their unique contributions to research without prejudice through the systematic collection of data on women in health research and ring-fencing of career development opportunities for women. Thirdly, institutions can establish communities of practice for women in global health research to cultivate their skills and confidence. Further, research in the area of inclusion is crucial to guiding the adoption of evidence-based practices to promote the participation and leadership of women in global health research.

Fourth, the commitment to the principles of equity and inclusion needs to be upheld by research leaders in innovative ways, beginning with increased intentionality in building a research workforce that reflects these principles. For instance, women researchers from LMICs have caring responsibilities and need to be supported to grow their careers without sacrificing these for unpaid caring roles. Organizations need to be supportive through flexible working arrangements and investments in staffing support to create a culture where women can actively participate in research. At the macro level, continued engagement of governments is necessary to enact childcare and social protection policies and programs that can contribute to closing the gender equity gap in research and create enabling environments for women in the workforce.

Lastly, it is time for global health to have more emerging women research leaders on a clearly defined research career trajectory. The research fraternity needs to push the boundaries by providing examples of best practices globally in applying these principles to redress the pain points in global health. There is not a one size fits all or prescriptive strategy, and our aspiration is that there is an emergence of multiple exemplars globally. We challenge various actors to move beyond the rhetoric and practically demonstrate to the world what good looks like for addressing the systemic barriers women in research face.

\section{THE LANGUAGE BARRIER}

Perhaps the fundamental hurdle most LMIC researchers face is that most are not native speakers of the languages in which research is most commonly published. This fact immediately puts them at a disadvantage when crafting effective communications. This is reflected across grant applications, manuscripts preparation and oral presentations, which are required to advance their careers. ${ }^{6}$ LMICs academic institutions should sensitize their governments on the importance of allocating funding to train future researchers with a full array of research skills such as proficiency in manuscript writing and copy editing. Academic institutions should work with research organizations and various government ministries to place their students for research internships to hone these skills. LMICs governments and especially the ministries of education must have the political will to embed a culture of research through workshops, providing funding for early career researchers to attend international conferences, and creating exchange research partnerships with HICs universities.

Similarly, journal editors need to intentionally not overlook the crucial contributions from LMIC researchers that may be poorly presented. Strategies to be adopted include providing free editorial services for non-English speakers and the translation manuscript databases where authors can write in their language well understood by the local people who are users of this research. Chiefly, it should never be forgotten that LMIC researchers investigating the conditions and problems of their societies have a tremendous advantage: they know their societies from the inside out. If the world is to profit from this essential value-added, 
editors need to take extra steps to encourage promising local researchers to improve their writing skills. Helpful interventions might include programs like the Pre-Publication Support Service, a non-profit initiative run by the University of Michigan, ${ }^{6}$ which can be replicated and extended to young writers seeking to publish in first-rank peer-reviewed journals.

\section{CONCLUSION}

Can we redress these imbalances? We posit that it is possible, although there must be a genuine commitment to do so. Establishing public review mechanisms to gauge how well the global health research stakeholders meet these standards is critical. In addition, journal editors need to be sensitized to consider inclusiveness as a metric for engagement with local stakeholders in LMICs communities where data is collected. Inclusiveness will ensure that researchers from LMICs have the opportunity to narrate their side of the story. As the old African adage goes, 'Until the lion learns how to write, every story will glorify the hunter.' We are at the cusp of redefining an equitable global health research landscape, and we all have a collective role to play. Are we ready?

\section{FUNDING}

The author(s) received no financial support for the authorship, and/or publication of this article.

\section{AUTHORSHIP CONTRIBUTIONS}

Both authors developed and contributed equally to this manuscript.

\section{COMPETING INTERESTS}

We declare no competing interests.

\section{CORRESPONDENCE TO:}

Dr. Constance S Shumba, School of Nursing and Midwifery, Aga Khan University, Kenya, P.O. Box 39340-00623, Nairobi, Kenya. +254(0)714713908.

Dr. Constance S Shumba (PhD), is a global health practitioner and researcher trained in the UK who has worked in various parts of sub-Saharan Africa and Asia. She is a faculty member at the School of Nursing and Midwifery, Aga Khan University, Kenya.

Dr. Adelaide M Lusambili, Department of Population Health, Medical College, Aga Khan University, P.O. Box 30270-00100, Nairobi, Kenya. +254(0)700920089. E-mail: adelaide.lusambili@aku.edu

Dr. Adelaide M Lusambili (PhD), is a Social Behavioral Research Scientist trained in the US and has conducted research and mentored students in the UK and Kenya. Currently, she is a faculty member at the Department of Population Health, Medical College, Aga Khan University.

Submitted: July 07, 2021 CET, Accepted: July 15, 2021 CET 


\section{REFERENCES}

1. Dimitris MC, Gittings M, King NB. How global is global health research? A large-scale analysis of trends in authorship. BMJ Glob Health.

2021;6(1):e003758. doi:10.1136/bmigh-2020-003758

2. Boum Y II, Burns BF, Siedner M, Mburu Y, Bukusi E, Haberer JE. Advancing equitable global health research partnerships in Africa. BMJ Glob Health. 2018;3(4):e000868. doi:10.1136/bmigh-2018-000868

3. Tong A, Sainsbury P, Craig J. Consolidated criteria for reporting qualitative research (COREQ): a 32-item checklist for interviews and focus groups. International Journal for Quality Health Care. 2007;19(6):349-357. doi:10.1093/intahc/mzm042
4. Tricco AC, Lachance CC, Rios P, et al. Global evidence of gender inequity in academic health research: a living scoping review protocol. JBI Evidence Synthesis. 2020;18(10):2181-2193. doi:10.111 24/ibies-20-00078

5. Abimbola S, Pai M. Will global health survive its decolonisation? Lancet. 2020;396(10263):1627-1628. doi:10.1016/s0140-6736(20)32417-x

6. Busse C, August E. Addressing power imbalances in global health: Pre-Publication Support Services (PREPSS) for authors in low-income and middleincome countries. BMJ Glob Health. 2020;5(2):e002323. doi:10.1136/bmigh-2020-002323 